\title{
An early experience with Coronary Artery Bypass Surgery: Is there a learning curve for Off Pump Coronary Artery Bypass technique?
}

\author{
Ramesh R Koirala, Siddhartha Pradhan, Anil Acharya, Pramod Sharma, \\ Bhagawan Koirala.
}

Ischemic heart disease is a major cause of morbidity and mortality even in Nepal. Data from various clinical trials and retrospective studies suggest that as the number of diseased major coronary arteries increases, the greater the survival benefit of surgical therapy over medical therapy alone.

In our Shahid Gangalal National Heart Center we started performing Coronary Artery Bypass grafting from 2002. By 2004 January, we had done Coronary Artery Bypass grafting on a total of 54 Patients. From September 2003 we started performing Off Pump Coronary Artery surgery (OPCAB) and by November 2003 we are doing exclusively OPCAB. Among them 36 patients undergone on-pump coronary grafting (CABG), 3 patients had on-pump beating heart CABG and next 15 patients had off pump beating heart (OPCAB) coronary bypass surgery.

There were total 46 male and 8 female (Male: Female ratio was 5,7:1). 35 (64.9\%) patients had recent Myocardial infarction, 9 (16.7\%) patients had Unstable angina and $11(20.4 \%)$ patients had Stable angina, The common coronary risk factors were male sex and hypertension (77\%). Among all these patient $33.3 \%$ had impaired LV ejection fraction. Tripple vessel disease was prevalent in these patients $(59.3 \%)$ in comparison to single vessel disease $(13.0 \%)$ and double vessel disease $(27.8 \%)$. Three or more vessel bypass was common in this series (53.7\%), LIMA was used in all but one cases $(98.1 \%)$ where as unilateral Radial artery was used in $27.8 \%$ of cases, In CPB-CABG group mean cardiopulmonary bypass time was 104.2 minutes (range 35 minutes to185 minutes) and aortic cross clamp time was 57.8 minutes (16 minutes-118 minutes), The mean postoperative bleeding was $743 \mathrm{ml}$ in first 24 hours $(70 \mathrm{ml}-3700 \mathrm{ml})$, Reexploration was done in one. The mean ICU stay was 3.09 days (1days- 16 days) where as mean postoperative hospital stay was 13.8 days (2days-140days), The most common postoperative complication was superficial surgical wound infection (15\%). 
We also compared age, sex, preoperative LV ejection fraction, postoperative bleeding, ICU stay, hospital stay and mortalily between OPCAB group and CPB- CABG group. Details of this will be discussed.

We conclude from this review OPCAB seems to have superiority than CPB- CABG in terms of shorter ICU stay and shorter hospital stay with same operative risk. 